\title{
Diagnostic Study of Nickel Plasma Produced by Fundamental (1064 nm) and Second Harmonics (532 nm) of an Nd: YAG Laser
}

\author{
M. Hanif ${ }^{1}$, M. Salik ${ }^{2,3}$, M. A. Baig ${ }^{2}$ \\ ${ }^{1}$ MCS, National University of Sciences \& Technology, Rawalpindi, Pakistan \\ ${ }^{2}$ National Center for Physics, Quaid-i-Azam University Campus, Islamabad, Pakistan \\ ${ }^{3}$ School of Science, Jiaaotong University, Beijing, China \\ Email: drhanif-mcs@nust.edu.pk
}

Received August 23, 2012; revised September 23, 2012; accepted September 30, 2012

\begin{abstract}
In the present work, we have studied the spatial evolution of the nickel alloy plasma produced by the fundamental (1064 $\mathrm{nm})$ and second $(532 \mathrm{~nm})$ harmonics of a Q-switched Nd: YAG laser by placing the target material in air at atmospheric pressure. The four Ni I lines at $335.10 \mathrm{~nm}, 394.61 \mathrm{~nm}, 481.19 \mathrm{~nm}$ and $515.57 \mathrm{~nm}$ are used for the determination of electron temperature $\left(T_{e}\right)$ using Boltzmann plot method. The electron temperature is calculated as a function of distance from the target surface for both modes of Nd: YAG laser. In case of fundamental (1064 nm) mode of laser, the temperature varies from $13700-10270 \mathrm{~K}$ as the distance is varied from 0 to $2 \mathrm{~mm}$. Whereas, in the case of second (532 nm) mode of laser it varies from $13270-9660 \mathrm{~K}$ for the same distance variation. The electron temperature has also been determined by varying the energy of the laser from 90 to $116 \mathrm{~mJ}$, for the fundamental (1064 nm) harmonic and from 58 to $79 \mathrm{~mJ}$ for the second $(532 \mathrm{~nm})$ harmonics of the laser. The temperature increases from 14192 to $15765 \mathrm{~K}$ in the first case and from 13,170 to $14,800 \mathrm{~K}$ for the second case. We have also studied the spatial behavior of the electron number density in the plasma plume. The electron number density $\left(N_{e}\right)$ in the case of fundamental $(1064 \mathrm{~nm})$ harmonic of the laser having pulse energy $125 \mathrm{~mJ}$ varies from $2.81 \times 10^{16}$ to $9.81 \times 10^{15} \mathrm{~cm}^{-3}$ at distances of $0 \mathrm{~mm}$ to $2.0 \mathrm{~mm}$, whereas, in the case of second $\left(532 \mathrm{~nm}\right.$ ) harmonic, with pulse energy $75 \mathrm{~mJ}$ it varies from $3.67 \times 10^{16}$ to $1.48 \times 10^{16} \mathrm{~cm}^{-3}$ for the same distance variation by taking $\mathrm{Ni}$ I line at $227.20 \mathrm{~nm}$ in both the cases.
\end{abstract}

Keywords: Laser Plasma; Laser Induced Breakdown Spectroscopy (LIBS); Electron Temperature and Electron Number Density

\section{Introduction}

In the present study, Laser induced breakdown spectroscopy (LIBS) has been employed, which is an analytical promising detection technique for solid, liquid and gaseous samples and is based on optical detection of certain atomic and molecular species by monitoring their emission signals from the laser induced plasma. This technique is very simple as compared to many other types of elemental analysis methods because of its straightforward experimental set-up. In it, one requires a pulsed laser for generating micro plasma on the target surface and the elemental analysis is accomplished by studying the emission of the plasma plume. The nature and dynamic of the laser induced plasma depends on different parameters such as, laser wavelength, spot size, pulse width and ambient environment etc. By using this technique, experiments can be performed either in air or in the presence of some ambient gas. During ablation proc- ess, laser energy is used in dissipation into the sample through heat conduction, melting and vaporization of the target material to generate plasma plume [1-5]. The element of nickel being good metal and vast applications in engineering remained under research since long. Moreover, after the invention of LASER, many researchers studied it by focusing on various aspects of interest [613].

In the present work, we have used LIBS technique to study the spatial evolution of the nickel plasma generated by the fundamental $(1064 \mathrm{~nm})$ and second $(532 \mathrm{~nm})$ harmonics of a Q-switched Nd: YAG laser. The experimentally observed line profiles of neutral nickel (Ni I) have been used to extract the electron temperature using the Boltzmann plot method. Whereas, the electron number density has been determined from the Stark broadening. Beside we have studied the variation of electron temperature and electron number density as a function of 
laser energy.

\section{Experimental Details}

The schematic diagram of experimental system as shown in Figure 1 is same as described in our previous work [14-16]. Briefly we used a Q-switched Nd: YAG (Quantel Brilliant) pulsed laser having pulse duration of $5 \mathrm{~ns}$ and $10 \mathrm{~Hz}$ repetition rate which is capable of delivering $400 \mathrm{~mJ}$ at $1064 \mathrm{~nm}$, and $200 \mathrm{~mJ}$ at $532 \mathrm{~nm}$. The laser pulse energy was varied by the flash lamp Q-switch delay through the laser controller, and the pulse energy was measured by a Joule meter (Nova-Quantel 01507). The laser beam was focused on the target using convex lens of $20 \mathrm{~cm}$ focal length. The sample was mounted on a three dimensional sample stage, which was rotated to avoid the non-uniform pitting of the target. The distance between the focusing lens and the sample was kept less than the focal length of the lens to prevent any breakdown of the ambient air in front of the target. The spectra were obtained by averaging 10 data of single shot under identical experimental conditions. The radiation emitted by the plasma were collected by a fiber optics (high-OH, core diameter: $600 \mu \mathrm{m})$ having a collimating lens $\left(0^{\circ}\right.$ $45^{\circ}$ field of view) placed at right angle to the direction of the laser beam. The optical fiber was connected with the LIBS-2000 detection system (Ocean Optics Inc.), to measure the plasma emission. The emission signal was corrected by subtracting the dark signal of the detector through the LIBS software. The LIBS-2000 detection system is equipped with five spectrometers each having slit width of $5 \mu \mathrm{m}$, covering the range between 220 - 720 nm. Each spectrometer has 2048 element linear CCD array and an optical resolution of $\approx 0.05 \mathrm{~nm}$ by scanning a narrow bandwidth dye laser. In the experiments, the time delay between the laser pulses and the start of the data acquisition is about $3.5 \mu \mathrm{s}$, whereas the system inte-

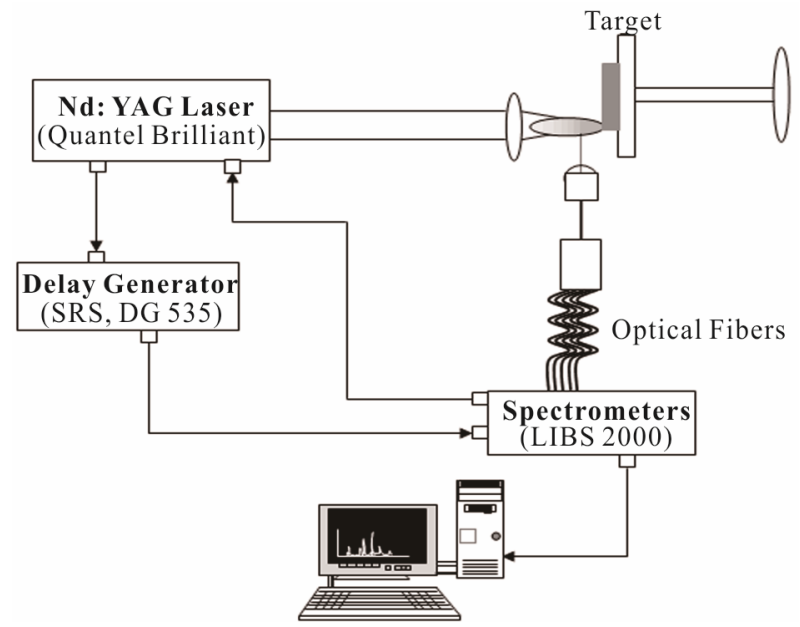

Figure 1. Schematic diagram of experimental set up. gration time is $2.1 \mathrm{~ms}$. In order to record the emission spectrum, the LIBS-2000 detection system was synchronized with the Q-switch of the Nd: YAG laser. The flash lamp out of the Nd: YAG laser triggered detection system through a four-channel digital delay/Pulse generator (SRS DG 535). The LIBS-2000 detection system triggered the Q-switch of the Nd: YAG laser.

\section{Results and Discussion}

\subsection{Optical Emission Spectra}

In the present work, we have produced nickel alloy plasma using fundamental (1064 $\mathrm{nm}$ ) and second (532 $\mathrm{nm})$ harmonics of a Q-switched Nd: YAG laser. In the first set of experiments, the fundamental (1064 nm) laser having $400 \mathrm{~mJ}$ pulse energy and $5 \mathrm{~ns}$ pulse width was focused on the target placed in the air at atmospheric pressure. The emission spectra of the plasma produced at the surface of the target is recorded at different distances along the direction of expansion of the plume. The ground state configuration of nickel is $3 d^{8}\left({ }^{3} \mathrm{~F}\right) 4 s^{2}$ which yields several levels.

In the Figure 2, we show the emission spectrum of $\mathrm{Ni}$ alloy plasma covering the spectral region from 320 to $400 \mathrm{~nm}$. The lines at $324.84,335.10$ and $394.61 \mathrm{~nm}$ belong to neutral nickel and are identified as $3 d^{9}\left({ }^{2} \mathrm{D}\right) 4 \mathrm{~s} \rightarrow$ $3 d^{8}\left({ }^{3} \mathrm{~F}\right) 4 s 4 p\left({ }^{3} \mathrm{P}^{\circ}\right), 3 d^{8}\left({ }^{3} \mathrm{~F}\right) 4 s^{20} \rightarrow 3 d^{8}\left({ }^{3} \mathrm{~F}\right) 4 s 4 p\left({ }^{3} \mathrm{P}^{\circ}\right)$ and $3 d^{8}$ $\left({ }^{3} \mathrm{~F}\right) 4 s^{2} \rightarrow 3 d^{8}\left({ }^{3} \mathrm{~F}\right) 4 s 4 p\left({ }^{3} \mathrm{P}^{\circ}\right)$ respectively.

In Figure 3, we show the emission spectrum of $\mathrm{Ni}$ alloy plasma covering the spectral region from 450 to 485 $\mathrm{nm}$. All observed transition lines in this region also belong to neutral nickel and iron. The dominating lines at 468.62, 472.92, and $481.19 \mathrm{~nm}$ belong to neutral nickel $\left(\mathrm{Ni}\right.$ I) and are identified as $3 d^{8}\left({ }^{3} \mathrm{~F}\right) \quad 4 \mathrm{~s} 4 \mathrm{p}\left({ }^{3} \mathrm{P}^{\circ}\right) \rightarrow$ $3 d^{8} 4 s\left({ }^{4} \mathrm{~F}\right) 5 s, 3 d^{8}\left({ }^{3} \mathrm{~F}\right) 4 s 4 p\left({ }^{3} \mathrm{P}^{\circ}\right) \rightarrow 3 d^{8} 4 s\left({ }^{2} \mathrm{~F}\right) 5 s$ and $3 d^{9}\left({ }^{2} \mathrm{D}\right) 4 p \rightarrow 3 d^{8}\left({ }^{1} \mathrm{~S}\right) 4 s^{2}$ respectively.

All the observed lines in the investigated spectral re-

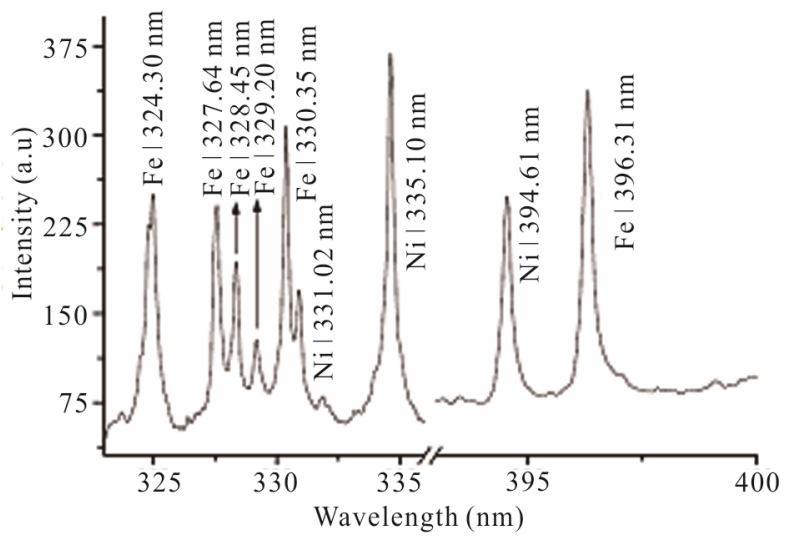

Figure 2. The emission spectrum of $\mathrm{Ni}$ alloy plasma generated by fundamental $(1064 \mathrm{~nm})$ harmonic of the laser covering the region from 320 to $400 \mathrm{~nm}$. 


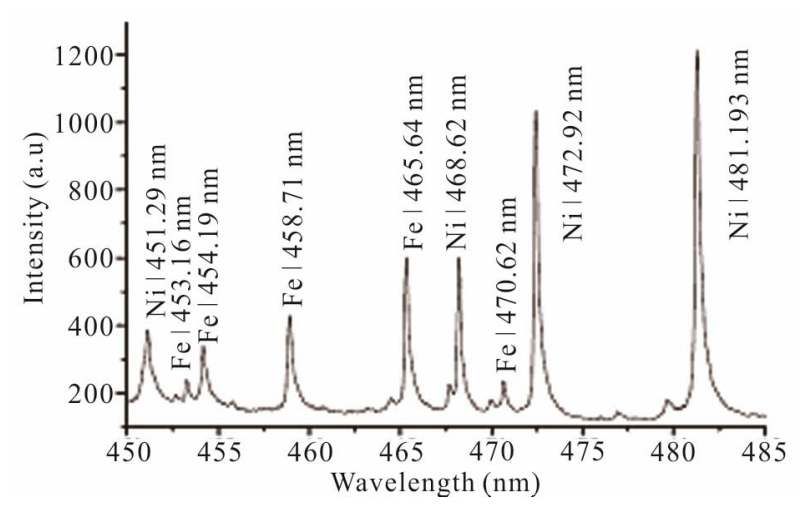

Figure 3. The emission spectrum of $\mathrm{Ni}$ alloy plasma generated the by fundamental $(1064 \mathrm{~nm})$ harmonic of the laser covering the region from 450 to $485 \mathrm{~nm}$.

gion along-with their assignments are listed in Table $\mathbf{1}$ (given at the end) based on the data given in the NBS Tables [17,18].

\subsection{Determination of Electron Temperature}

Plasma temperature is one of the most important properties of any excitation source, and its determination is important to understand the dissociation, ionization and excitation processes taking place in the plasma [19]. When the laser light interacts with the target surface, outer most electrons of the atoms get excited, and when the energy is greater than the binding energy of the target material, bond breaking occurs and evaporation of target material starts. The appearance of the plasma in front of the target changes the character of thermal and mechanical influence of laser radiation on the target. As the ionization potential of atoms is very large as compare to the laser energy, hence ionization is due to multi-photon ionization. The electron temperature is determined using the Boltzmann plot method from the relative intensities of the observed line, which are normally proportional to the population of the pertinent upper levels. The following relation has been used to extract the plasma temperature [20]:

$$
\ln \left(\frac{I_{k i} \lambda_{k i}}{A_{k i} g_{k}}\right)=\ln \left(\frac{N(T)}{U(T)}\right)-\frac{E_{k}}{k T}
$$

where, $I_{k i}$ is the integrated line intensity of the transition involving an upper level $(k)$ and a lower level $(i), \lambda_{k i}$ is the transition wavelength, $A_{k i}$ is the transition probability, $g_{k}$ is the statistical weight of level $(k), N(T)$ is the total number density, $U(T)$ is the partition function, $E_{k}$ is the energy of the upper level, $k$ is the Boltzmann constant and $T$ is the electron temperature. A plot of $\ln (\lambda I / g A)$ versus the term energy $E_{k}$ gives a straight line with a slope equal to $(-1 / k T)$. Thus the electron temperature can be determined without the knowledge of the total number density or the partition function. Errors are bound to be present in the determination of the electron temperature by this method therefore; the electron temperature is determined with $\approx 10 \%$ uncertainty, coming mainly from the transition probabilities and the measurement of the integrated intensities of the spectral lines. The line identifications and different spectroscopic parameters such as wavelength $(\lambda)$, statistical weight $(g)$, transition probability $(A)$ and term energy $(E)$ listed in the Table $\mathbf{1}$.

The four neutral nickel (Ni I) lines at 335.10, 394.61, 481.19 and $515.57 \mathrm{~nm}$ are used for the determination of electron temperature using Boltzmann plot method as shown in Figure 4. The electron temperature has been calculated as a function of distance from the target surface for both modes of the laser as shown in the Figure 5. In the case of fundamental $(1064 \mathrm{~nm})$ laser, the temperature varies from 13700 to $10270 \mathrm{~K}$ as the distance is varied from 0.05 to $2 \mathrm{~mm}$. Whereas, it varies from 13270 to $9660 \mathrm{~K}$ in the case of second $(532 \mathrm{~nm})$ harmonic of the laser over the same variation of the distance.

\subsection{Determination of Electron Number Density}

During the evolution of laser induced plasma (LIP), excitation and ionization of the evaporated material occur. It is then important to determine the thermodynamic parameters of LIP such as electron number density and

Table 1. Spectroscopic parameters of the Ni I lines.

\begin{tabular}{|c|c|c|c|c|c|c|c|}
\hline \multirow{2}{*}{$\mathrm{Sr}$} & \multirow{2}{*}{ Wavelength $\lambda(\mathrm{nm})$} & \multirow{2}{*}{ Transitions } & \multicolumn{2}{|c|}{ Statistical weight } & \multirow{2}{*}{$\begin{array}{c}\text { Transition } \\
\text { probability } A_{k i}\left(\mathrm{~s}^{-1}\right)\end{array}$} & \multicolumn{2}{|c|}{ Energy $\left(\mathrm{cm}^{-1}\right)$} \\
\hline & & & $g_{k}$ & $g_{i}$ & & $E_{k}$ & $E_{i}$ \\
\hline 1 & 227.20 & $3 d^{9}\left({ }^{2} \mathrm{D}\right) 4 s^{3} \mathrm{D}_{3} \rightarrow 3 d^{8}\left({ }^{3} \mathrm{P}\right) 4 s 4 p\left({ }^{3} \mathrm{P}^{\circ}\right)^{5} \mathrm{D}_{3}^{\circ}$ & 9 & 7 & $2.3 \times 10^{6}$ & 44206.099 & 204.787 \\
\hline 2 & 335.10 & $3 d^{8}\left({ }^{3} \mathrm{~F}\right) 4 s^{23} \mathrm{~F}_{4} \rightarrow 3 d^{8}\left({ }^{3} \mathrm{~F}\right) 4 s 4 p\left({ }^{3} \mathrm{P}^{\circ}\right)^{5} \mathrm{~F}^{\circ}{ }_{3}$ & 7 & 9 & $2.8 \times 10^{8}$ & 29832.779 & 0 \\
\hline 4 & 481.19 & $3 d^{9}\left({ }^{2} \mathrm{D}\right) 4 p^{3} \mathrm{P}^{0}{ }_{1} \rightarrow 3 d^{8}\left({ }^{1} \mathrm{~S}\right) 4 s^{21} \mathrm{~S}_{0}$ & 1 & 3 & $9.5 \times 10^{6}$ & 50276.321 & 29500.674 \\
\hline 5 & 515.57 & $3 d^{9}\left({ }^{2} \mathrm{D}\right) 4 p 1 \mathrm{D}_{2}^{\circ} \rightarrow 3 d^{9}\left({ }^{2} \mathrm{D}_{3 / 2}\right) 4 d^{2}(5 / 2)_{3}$ & 7 & 5 & $2.9 \times 10^{7}$ & 50832.001 & 31441.635 \\
\hline
\end{tabular}




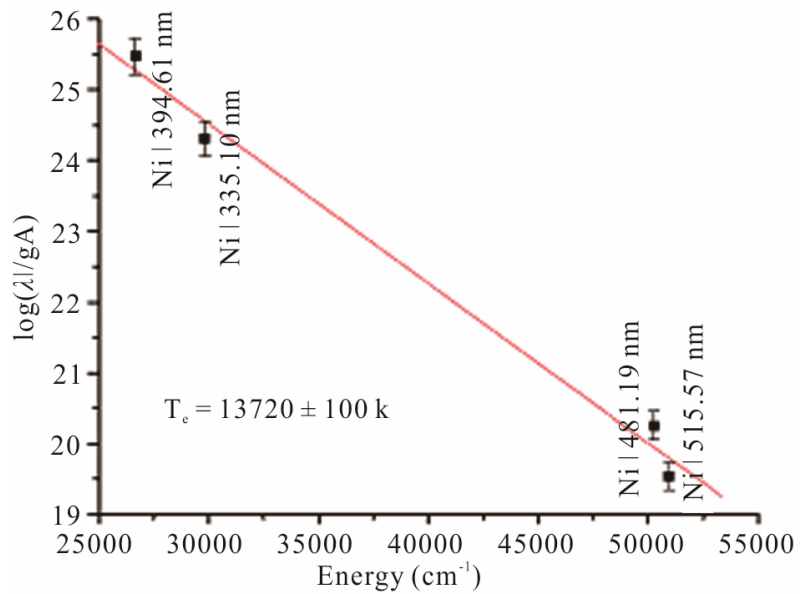

Figure 4. Boltzmann plot for the Ni I spectral lines at $\mathbf{0 . 0 5}$ $\mathrm{mm}$ from the target surface using fundamental $(1064 \mathrm{~nm})$ harmonic of the laser.

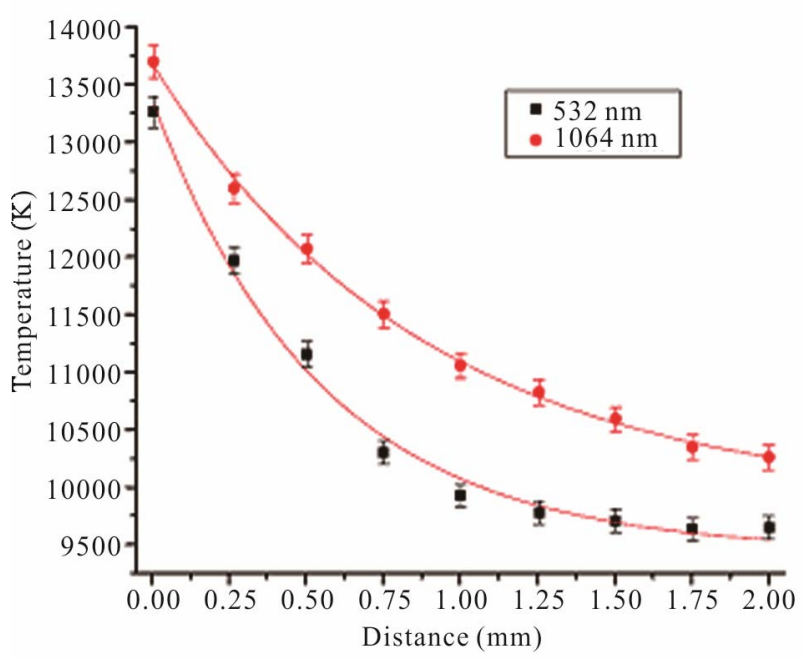

Figure 5. Variation of the electron temperature, along the direction of propagation of the plasma plume, using the fundamental $(1064 \mathrm{~nm})$ and second $(532 \mathrm{~nm})$ harmonics of the Nd: YAG laser.

electron temperature. One of the most reliable techniques to determine the electron number density is from the measured Stark broadened line profile of an isolated line of either neutral atom or single charge ion. The electron number density $\left(N_{e}\right)$, related to the full width at half maximum (FWHM) of the Stark broadening lines is given by the following relation $[3,5,20]$ :

$\Delta \lambda_{1 / 2}=2 \omega\left(\frac{N_{e}}{10^{16}}\right)+3.5 A\left(\frac{N_{e}}{10^{16}}\right)^{1 / 4}\left[1-\frac{3}{4} N_{D}^{-1 / 3}\right] \omega\left(\frac{N_{e}}{10^{16}}\right)$

where, $\omega$ is the electron impact width parameter, $A$ is the ion broadening parameter, $N_{e}$ is the electron number density and $N_{D}$ is the number of particles in the Debye sphere. The first term in Equation (2) refers to the broadening due to the electron contribution, whereas, the second term is attributed to the ion broadening. Since the contribution of the ionic broadening is normally very small, therefore, it can be neglected. The electron number densities have been determined from the line profiles of the isolated nickel neutral line at $227.20 \mathrm{~nm}$ using the relation (3) by neglecting the contribution of the ion impact broadening and Doppler broadening in a relation (2):

$$
\Delta \lambda_{1 / 2}=2 \omega\left(\frac{N_{e}}{10^{16}}\right)
$$

The value of $\omega$ corresponding to different electron temperatures is obtained from the reference data [13].

In the Figure 6, we show the line profile of the neutral nickel line at $515.57 \mathrm{~nm}$ recorded from the plasma generated by the second harmonic $(532 \mathrm{~nm})$ of the laser. The laser energy was varied from 52 to $75 \mathrm{~mJ}$ for the various corresponding values of Q switch delay from 10 to $80 \mu \mathrm{s}$. The width of the line profile increases as the laser energy is increased and its value is maximum at $40 \mu$ s delay.

In the Figure 7, we show the Stark broadened profile of neutral nickel line at $335.10 \mathrm{~nm}$ recorded from the plasma using the first harmonic $(1064 \mathrm{~nm})$ of the laser. The full line represents the Lorentizian fit to the experimental data points. The full width half maxima (FWHM) of the spectra are used to estimate the electron number density. The spatial behavior of the electron number density in the plume is determined using the above relation 3.

The electron number density $\left(N_{e}\right)$ in the case of fundamental harmonic $(1064 \mathrm{~nm})$ of the laser having pulse energy $125 \mathrm{~mJ}$ varies from $2.81 \times 10^{16}$ to $9.81 \times 10^{15} \mathrm{~cm}^{-3}$

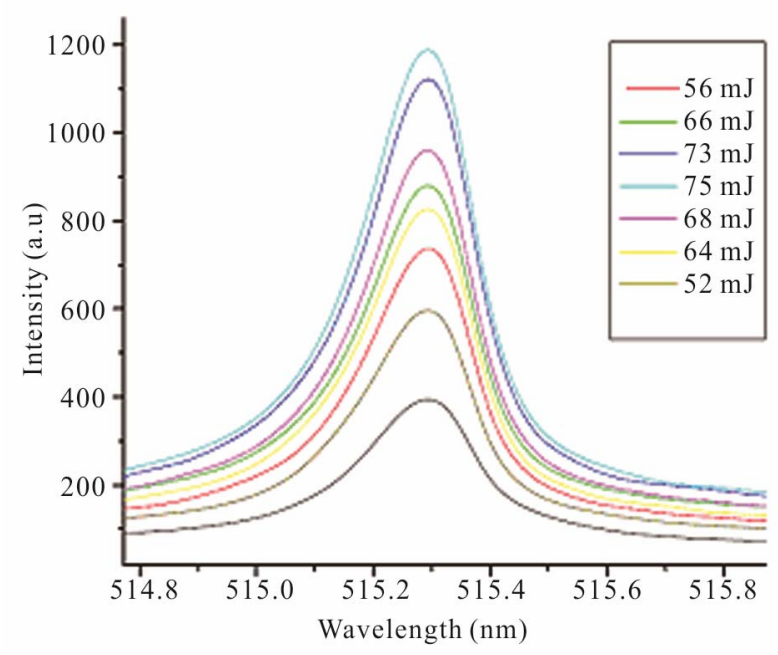

Figure 6. (Color line) Variation in the signal intensity and width of the neutral nickel line at $515.57 \mathrm{~nm}$ using second harmonic $(532 \mathrm{~nm})$ of the Nd: YAG laser. 


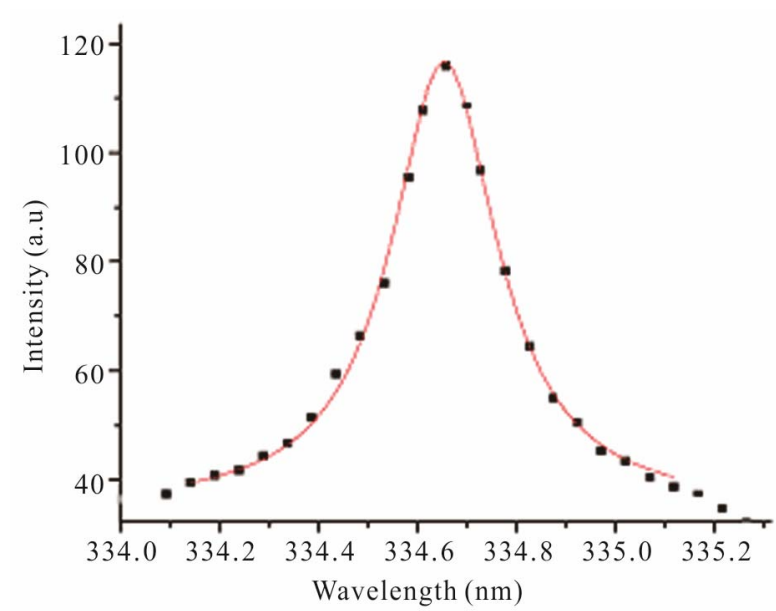

Figure 7. Stark broadening profile of $\mathrm{Ni} I$ line at $335.10 \mathrm{~nm}$. The dots represent the experimental profile and the solid line is Lorentizion fit.

at distances of 0.05 to $2.0 \mathrm{~mm}$ as shown in the Figure 8 .

In the case of second harmonic $(532 \mathrm{~nm})$ of the laser with pulse energy $75 \mathrm{~mJ}$ it varies from $3.67 \times 10^{16}$ to $1.48 \times 10^{16} \mathrm{~cm}^{-3}$ at a distance of 0.05 to $2.0 \mathrm{~mm}$ from the target surface by taking neutral nickel line at $227.20 \mathrm{~nm}$ in both the cases. It is evident that the electron number density is higher for second harmonic (532 nm) as compared to first harmonic $(1064 \mathrm{~nm})$ of $\mathrm{Nd}$ : YAG laser, which demonstrates that the mass ablation rate is maximum for the shorter wavelength laser. It is observed that electron number density close to the target surface $(0.05$ $\mathrm{mm}$ ) is maximum and decreases as the distance from the target is increased. The electron temperature and electron number density are both maximum close to the target surface $(0.05 \mathrm{~mm})$, since the region close to the surface continuously absorbs the laser radiation during the laser pulse. When the plasma expands it thermalizes by transferring the energy to its surroundings. Moreover, it is transparent to the laser pulse; therefore, the electron temperature and the electron number density decrease along the direction of expansion of the plume. The electron temperature and electron number density are different for the two modes of the Nd: YAG laser, because of the difference in the energy per photon in each mode.

\subsection{Variation of Plasma Parameters}

In the second set of experiments, we have determined the electron temperature $\left(T_{e}\right)$ and electron number density $\left(N_{e}\right)$ for different values of the laser energy by using both modes of the Nd: YAG laser at 1064 and $532 \mathrm{~nm}$ wavelengths. We have observed that the intensities and widths of the spectral lines increase with the increase in the laser energy. The electron temperature has also been determined by varying the energy of the laser from 90 to 116 $\mathrm{mJ}$, for the fundamental $(1064 \mathrm{~nm})$ harmonic and from

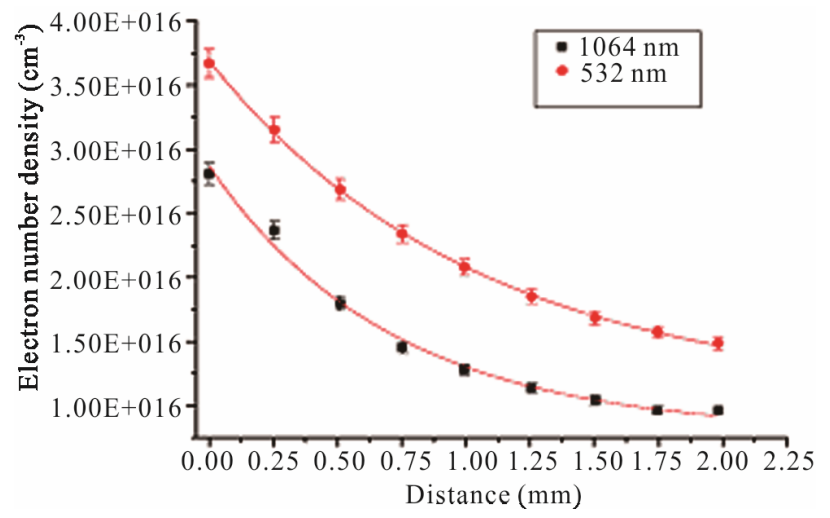

Figure 8. (Color line) Variation of the electron number density with the distance using the fundamental $(1064 \mathrm{~nm})$ and second $(532 \mathrm{~nm}$ ) harmonics of the Nd: YAG laser.

58 to $79 \mathrm{~mJ}$ for the second harmonic $(532 \mathrm{~nm})$ of the laser. The temperature increases from 14192 to $15765 \mathrm{~K}$ in the first case and from 13170 to $14800 \mathrm{~K}$ for the second case as shown in the Figures 9(a) and (b).

The electron temperature near the target surface is found to be higher and it increases with the wavelength, which is likely to be resulted from higher laser plasma energy transfer. Since the region near the surface of the target material constantly absorbs radiation during the time interval of the laser pulse, causing a higher temperature near the target surface. Decrease in electron temperature is due to the fact that the thermal energy is rapidly converted into kinetic energy when the plasma is attaining maximum expansion velocities, causing the temperature to drop for the expanding plasma.

In the Figures 10(a) and (b), we show the variation in the electron number density as a function of the laser energy. In case of fundamental harmonic (1064 nm) of the laser, with the variation of laser energy from 110 to $122 \mathrm{~mJ}$, the corresponding electron number densities varies from $1.83 \times 10^{15}$ to $1.54 \times 10^{16} \mathrm{~cm}^{-3}$. Whereas, in case of second harmonic $(532 \mathrm{~nm})$ of the laser, with the variation of laser energy from 49 to $70 \mathrm{~mJ}$, the corresponding electron number densities varies from $9.6 \times$ $10^{15}$ to $1.2 \times 10^{16} \mathrm{~cm}^{-3}$. The observed increase in $N_{e}$ and $T_{e}$ by the increase of the laser energy is due to the absorption and/or reflection of the laser photon by the plasma, which depends upon the plasma frequency. In our experiment, for both modes of the laser, the corresponding frequencies are $2.8 \times 10^{14}$ and $5.6 \times 10^{14} \mathrm{~Hz}$ respectively, whereas the plasma frequency is $v_{\mathrm{p}}=8.9 \times$ $10^{3} \sqrt{ } \mathrm{Ne}$. The electron number density is $N_{e} \approx 10^{16} \mathrm{~cm}^{-3}$, therefore, $v_{p}=3.6 \times 10^{12} \mathrm{~Hz}$ which is less then the laser frequency $\left(\approx 10^{14} \mathrm{~Hz}\right)$, which shows that, the energy loss due to the reflection of the laser from the plasma is insignificant. The use of the emission spectroscopy for the measurement of the temperature and electron number density requires optically thin spectral lines. The self ab- 


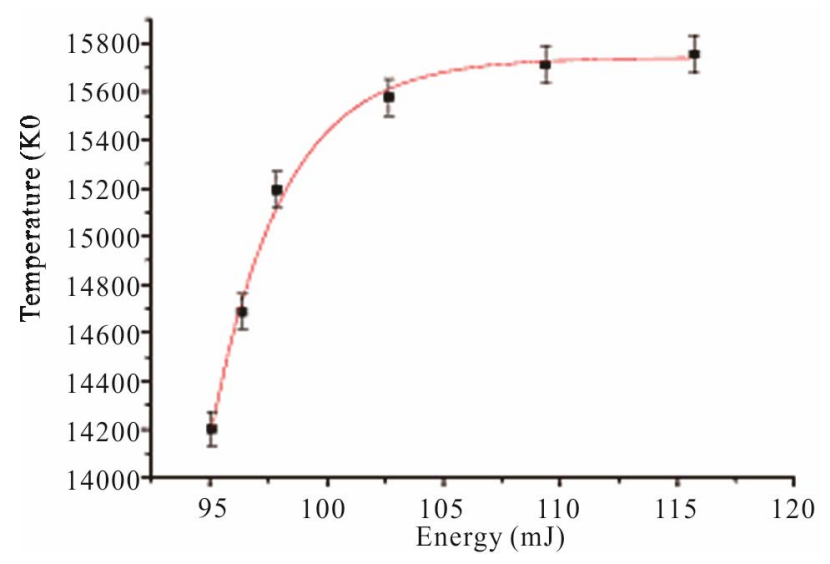

(a)

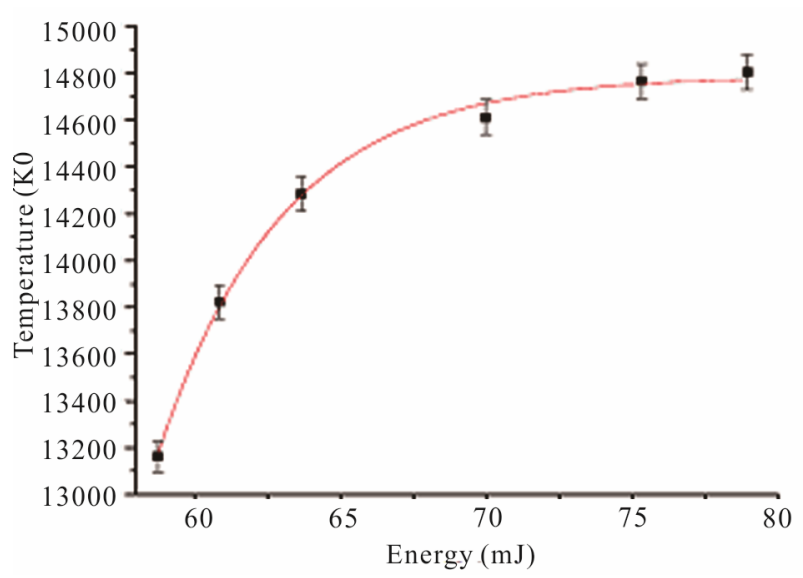

(b)

Figure 9. (a) Variation of the electron temperature $\left(T_{e}\right)$ with the laser energy using fundamental $(1064 \mathrm{~nm})$ harmonic of the Nd: YAG laser; (b) Variation of the electron temperature $\left(T_{e}\right)$ with the laser energy using second harmonic (532 nm) of the Nd: YAG laser.

sorption depends on the oscillator strength, level energies degeneracy, broadening parameters and also on the plasma parameters. The Ni-Fe alloy plasma is observed to be optically thin as in case of self absorption a strong line appears to have a dip at the central frequency (self absorption).

\subsection{Validity of Local Thermodynamic Equilibrium Condition}

The use of the emission spectroscopy for the determination of the electron temperature and electron number density requires optically thin spectral lines. The self absorption depends on the oscillator strength, level energies degeneracy, broadening parameters and also on the plasma parameters. The Ni alloy plasma is observed to be optically thin as in case of self absorption a strong line appears to have a dip at the central frequency (self absorption). In the present work we did not find any dip at

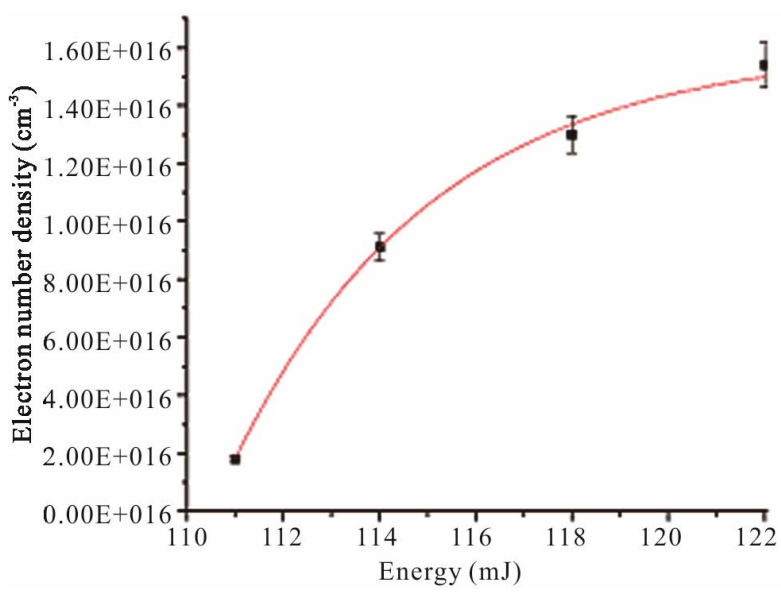

(a)

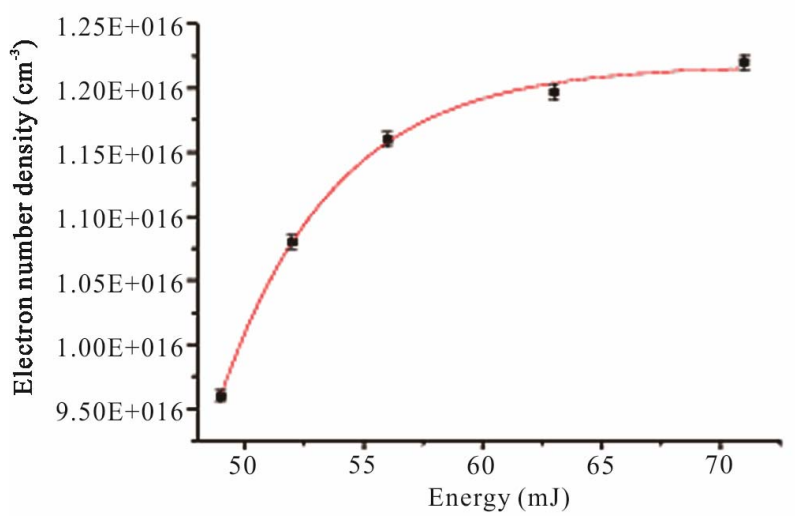

(b)

Figure 10. (a) Variation of the electron number density with the laser energy using the fundamental harmonic (1064 nm) of the Nd: YAG laser; (b) Variation of the electron number density with the laser energy using the second (532 $\mathrm{nm})$ harmonic of the Nd: YAG laser.

the central frequency of the observed emission lines. The condition that the atomic states should be populated and depopulated predominantly by electron collisions, rather than by radiation, requires an electron density which is sufficient to ensure the high collision rate. The corresponding lower limit of the electron density is given by Mc Whirter criterion, which is the condition for attaining the minimum number density to check the validity of the local thermodynamic equilibrium [3,21,22]:

$$
N_{e} \geq 1.6 \times 10^{12} \mathrm{~T}^{1 / 2}(\Delta E)^{3}
$$

where, $N_{e}\left(\mathrm{~cm}^{-3}\right)$ is the electron number density, $T(\mathrm{~K})$ is the electron temperature and $\Delta E(\mathrm{eV})$ is the difference in the energies between the upper and lower states of all the investigated transitions.

\section{Conclusion}

We have used a Q-switched Nd: YAG laser at its fundamental harmonic (1064 nm) and second harmonic (532 
$\mathrm{nm}$ ) to study the laser produced nickel alloy plasma. The emission spectrum of the plasma reveals transitions of neutral nickel and iron. The electron temperature and the electron number density have been determined along the axial positions of the plasma plume. The temperature and the electron number density both close to the target are maximum. The temperature and the number density decrease along the direction of expansion of the plume. The temperature and number density are different for both modes of the laser, because of the difference in the energy per photon in each mode. We have also determined the electron number density for different values of the laser energy. In both modes of the laser, we have observed an identical trend of the variation of electron number density as a function of the laser energy. The variation in the electron number density with the laser energy also shows a similar behaviour.

\section{Acknowledgements}

M. Hanif is thankful to MCS and National University of Sciences \& Technology (NUST), Islamabad for the encouragement in terms of provision of time and financial support to carry out research work.

\section{REFERENCES}

[1] L. J. Radziemski and D. A. Cremers, "Laser-Induced Plasmas and Applications," Marcel Dekker, New York, 1989.

[2] J. C. Miller and R. F. Haglund Jr., "Laser Ablation: Mechanisms and Applications," Springer-Verlag, Berlin, 1991. doi:10.1007/BFb0048346

[3] D. A. Cremers and L. J. Radziemski, "Handbook of Laser-Induced Breakdown Spectroscopy," Wiley, New York, 2006. doi:10.1002/0470093013

[4] A. Miziolek, V. Palleschi and I. Schechter, "Laser-Induced Breakdown Spectroscopy (LIBS): Fundamentals and Applications," Cambridge University Press, Cambridge, 2006. doi:10.1017/CBO9780511541261

[5] J. P. Singh and S. N. Thakur, "Laser-Induced Breakdown Spectroscopy,” Elsevier Science, Amsterdam, 2007.

[6] J. E. Lawler and S. Salih, "Radiative Lifetimes in Ni II," Physical Review A, Vol. 35, No. 12, 1987, pp. 5046-5050. doi:10.1103/PhysRevA.35.5046

[7] F. S. Ferrero, J. Manrique, M. Zwegers and J. Campost, "Determination of Transition Probabilities of $3 d^{8} 4 p-3 d^{8} 4 s$ Lines of Ni II by Emission of Laser-Produced Plasmas," Journal of Physics B: Atomic Molecular Physics, Vol. 30, No. 4, 1997, pp. 893-903. doi:10.1088/0953-4075/30/4/011

[8] A. Varga, R. M. G. Martinez, G. Zaray and F. Fodor, "Investigation of Effects of Cadmium, Lead, Nickel and Vanadium Contamination on the Uptake and Transport Processes in Cucumber Plants by TXRF Spectrometry," Spectrochemica Acta Part B, Vol. 54, No. 10, 1999, pp. 14551462. doi:10.1016/S0584-8547(99)00105-6

[9] C. Aragon, J. Bengoechea and J. A. Aguilera, "Influence of the Optical Depth on Spectral Line Emission from Laser-Induced Plasmas," Spectrochemica Acta Part B, Vol. 56, No. 2, 2001, pp. 619-628. doi:10.1016/S0584-8547(01)00172-0

[10] K. Wagatsuma and H. Honda, "Influence of the Optical Depth on Spectral Line Emission from Laser-Induced Plasmas," Spectrochemica Acta Part B, Vol. 60, No. 12, 2005, pp. 1538-1544. doi:10.1016/j.sab.2005.10.004

[11] C. Aragon, F. Penalba and J. A. Aguilera, "Spatial Distributions of the Number Densities of Neutral Atoms and Ions for the Different Elements in a Laser Induced Plasma Generated with a Ni-Fe-Al Alloy," Analytical and Bioanalytical Chemistry, Vol. 385, No. 2, 2006, pp. 295-302. doi:10.1007/s00216-006-0301-0

[12] S. Nakamura and K. Wagatsuma, "Emission Characteristics of Nickel Ionic Lines Excited by Reduced-Pressure Laser-Induced Plasmas Using Argon, Krypton, Nitrogen, and Air as the Plasma Gas," Spectrochemica Acta Part B, Vol. 62, No. 9, 2007, pp. 1303-1310. doi:10.1016/j.sab.2007.10.008

[13] R. Mayo, M. Ortz and M. Plaza, "Measured Stark Widths of Several Ni II Spectral Lines," Journal of Physics B: Atomic Molecular and Optical Physics, Vol. 41, No. 9, 2008, pp. 1-5. doi:10.1088/0953-4075/41/9/095702

[14] M. Hanif, M. Salik and M. A. Baig, "Spectroscopic Studies of the Laser Produced Lead Plasma," Journal of Plasma Science and Technology, Vol. 13, No. 2, 2011, pp. 129-134. doi:10.1088/1009-0630/13/2/01

[15] M. Salik, M. Hanif and M. A. Baig, "Plasma Diagnostic Study of Alumina $\left(\mathrm{Al}_{2} \mathrm{O}_{3}\right)$ Generated by the Fundamental and Second Harmonics of a Nd:YAG Laser," IEEE Transactions on Plasma Science, Vol. 36, No. 9, 2011, pp. 1861-1866. doi:10.1109/TPS.2011.2159852

[16] M. Hanif, M. Salik and M. A. Baig, "Quantitative Studies of Copper Plasma Using Laser Induced Breakdown Spectroscopy," Journal of Optics and Lasers in Engineering, Vol. 49, No. 12, 2011, pp. 1456-1461. doi:10.1016/j.optlaseng.2011.06.013

[17] C. E. Moore, “Atomic Energy Levels," NBS Circular No. 467, Washington DC, 1971.

[18] NIST Atomic Spectra Database, "Kurucz Output Atomic Spectra Line Database from R.L. Kurucz's CD-ROM 23."

[19] D. Lacroix and G. Jeandel and C. Boudot, "Pectroscopic Characterization of Laser-Induced Plasma Created during Welding with a Pulsed Nd:YAG Laser," Journal of Applied Physics, Vol. 81, 1997, pp. 6599-6606. doi: $10.1063 / 1.365198$

[20] H. R. Griem, "Principles of Plasma Spectroscopy," Cambridge University Press, Cambridge, 1997. doi: $10.1017 / \mathrm{CBO} 9780511524578$

[21] R. W. P. McWhirter, "Plasma Diagnostic Techniques," Academic Press, New York, 1965.

[22] O. Barthélemy, J. Margot, S. Laville, F. Vidal, M. Chaker, T. W. Johnston, B. Le Drogoff and M. Sabsabi, "Investigation of the State of Local Thermodynamic Equilibrium of a Laser-Produced Aluminum Plasma," Applied Spectroscopy, Vol. 59, No. 4, 2005, pp. 529-536. doi:10.1366/0003702053641531 